\title{
Flexible, polymer gated, AC-driven organic electroluminescence devices
}

\author{
Junwei Xu, David Carroll
}

Junwei Xu, David L. Carroll, "Flexible, polymer gated, AC-driven organic electroluminescence devices," Proc. SPIE 10362, Organic Light Emitting Materials and Devices XXI, 1036215 (25 August 2017); doi:

$10.1117 / 12.2272172$

Event: SPIE Organic Photonics + Electronics, 2017, San Diego, California, United States 


\title{
Flexible, polymer gated, AC-driven organic electroluminescence devices
}

\author{
Junwei Xu $\mathrm{u}^{\mathrm{a}, \mathrm{b}}$ and Prof. David L. Carroll ${ }^{\mathrm{a}, \mathrm{b} *}$ \\ ${ }^{a}$ Center for Nanotechnology and Molecular Materials, Wake Forest University, Winston-Salem, NC,

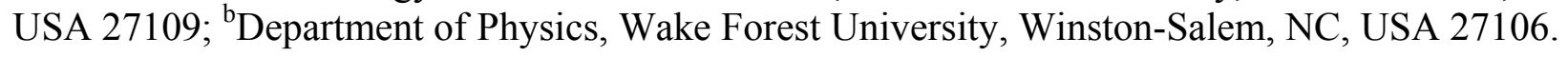

\begin{abstract}
Comparing rigid inorganic layer, polymeric semiconducting gate layer exhibits superior flexibility as well as efficient carrier manipulation in high frequency AC cycles. Mechanism of the carrier manipulation at the gate in forward and reversed bias of AC cycle is studied. The flexible PET-based AC-OEL device with poly[(9,9-bis(3'-((N,N-dimethyl)-Nethylammonium)-propyl)-2,7-fluorene)-alt-2,7-(9,9-dioctylfluorene)] (PFN-Br) gate shows a stable electroluminescent performance in frequency sweep with a color rendering index (CRI) over 81 at $2800 \mathrm{~K}$ color temperature.
\end{abstract}

Keywords: electroluminescence, alternating current (AC), polymeric carrier gate, white emission

\section{INTRODUCTION}

Form-factor advantages on luminescent performance have brought field induced electroluminescence (EL) device tremendous attentions for the sun-spectra, roll-type, indoor illumination source. ${ }^{1-4}$ Compared with conventional field induced EL device cooperating symmetric ${ }^{5,6}$ or asymmetric ${ }^{7-10}$ insulators which are sandwiching emitting complex, the AC driven organic EL (AC-OEL) device gated by n-type wide bandgap semiconducting layer exhibits efficient carrier manipulation and luminescent performance advances. ${ }^{11}$ By utilizing semiconductor gate between anode and hole generation layer (HGL), the direct hot hole injection is avoided in the negative half of AC cycle, meanwhile electron extraction is facilitated. Fully realized flexible AC-OEL device, however, is not achieved, which requires gate materials equip good mechanical performance with bending. For instant, polyvinylidene fluoride (PVDF) has been used in flexible AC-OEL devices ${ }^{12}$, however, its high dielectric constant normally results an high working voltage that can make the device lossy.

Here we present an AC-OEL structure that utilizes a polymeric semiconducting gate layer exhibiting state-of-the-art flexibility. The n-type polymer, PFN-Br, was demonstrated as ideal substitution of high-k dielectric layer in AC-OEL devices, serving the function of a "passive carrier management gate". With the optical design that takes a full advantage of emerging good-quality flexibility and efficient carrier manipulation, the proposed white PET-based AC-OEL device exhibits an advanced opto-electronic performance as well as superior flexibility.

*carrold1@,wfu.edu; phone 1336 758-5508; http://users.wfu.edu/carroldl/Welcome.html 


\section{METHODOLOGY}

\subsection{Materials and Device Fabrication}

The AC-OEL devices were fabricated on PET substrates with a pre-coated ITO film with a sheet resistance of $\approx 60 \Omega$ $\square^{-1}$. The substrates were cleaned in an ultrasonic bath and subsequently were dried in a vacuum oven for $2 \mathrm{~h}$ and treated with UV-ozone for $15 \mathrm{~min}$. A methanol solution of PFN-Br was spin-coated on the ITO substrate at $2000 \mathrm{rpm}$ for $30 \mathrm{~s}$ and then annealed at $100{ }^{\circ} \mathrm{C}$ for $15 \mathrm{~min}$ in vacuum oven. For inorganic gate layer, $\mathrm{ZnO}$ thin film was deposited using an RF sputter system (Kurt J. Lesker NANO 36). poly[N,N'-bis(4-butylphenyl)-N,N"- bis(phenyl)-benzidine] (poly-TPD) with $7 \mathrm{wt} \%$ 2,3,5,6-tetrafluoro-7,7,8,8tetracyanoquinodimethane (F4TCNQ) was dissolved in chlorobenzene and a hole generation layer was built in a nitrogen-filled glovebox. The emitting layers were obtained by spin-coating the $10 \mathrm{mg}$ $\mathrm{mL}^{-1}$ blend of poly(vinyl carbazole) (PVK) as a cohost and $2 \mathrm{wt} \%$ phosphorescent dyes, fac-tris(2-phenylpyridine)iridium(III) $\left[\operatorname{Ir}(\mathrm{ppy})_{3}\right]$ for green emission and iridium(III)bis(2-methyldibenzo[f,h]quinoxaline) (acetylacetonate) $\left[\operatorname{Ir}(\mathrm{MDQ})_{2}(\mathrm{acac})\right]$ for red emission, in chlorobenzene at $2000 \mathrm{rpm}$ and annealed on $100{ }^{\circ} \mathrm{C}$ hotplate for $30 \mathrm{~min}$. Next, the samples were transferred to a vacuum chamber at a pressure of about $2 \times 10^{-7}$ Torr for coating of a $30 \mathrm{~nm}$ thick electron injection layer (Bphen). Finally, a $10 \mathrm{~nm}$ layer of Ca and a $100 \mathrm{~nm}$ layer of $\mathrm{Al}$ were deposited via thermal evaporation with a rate of $2 \mathrm{As}^{-1}$ for top electrode.

\subsection{Materials and Device Fabrication}

A scanning electron microscope (SEM, JEOL 6330) was used to measure the top morphology of PFN-Br and $\mathrm{ZnO}$ on ITO-coated PET substrates after bending. The AC-OEL devices were measured in a nitrogen-filled glovebox at room temperature $\left(25^{\circ} \mathrm{C}\right.$ ) without sealing. A $200 \mathrm{MHz}$ function/arbitrary waveform generator (Agilent 33220A) connected to a Trek PZD700A M/S amplifier provides a sinusoidal signal with suitable voltage and frequency. An ILT 1400-A photometer (International Light Technologies) was used to measure the outcoupling luminance. EL spectra were collected using an ILT 950 spectroradiometer (International Light Technologies).

\section{RESULTS}

\subsection{Carrier manipulation at the gate}

Experimentally, we investigated current density for forward and reversed voltage of the gate structure: ITO/Gate/polyTPD: F4TCNQ/ $/ \mathrm{MoO}_{3} / \mathrm{Au}$. In Figure 1, the peak current of PFN-Br gate is $161.9 \mathrm{~mA} \mathrm{~cm}$ in the forward bias of $3 \mathrm{~V}$, and crosses through the origin. As the bias is reversed to $3 \mathrm{~V}$, the current becomes independent from the applied voltage, maintaining a low level constant (less than $0.67 \mathrm{~mA} \mathrm{~cm}{ }^{-2}$ ). This is indicative of the "gate off" condition in reverse bias. Comparing to $\mathrm{ZnO}$ gate, the PFN-Br gate device shows a higher current on-off ratio of 242. The high electron mobility makes wide band gap n-type semiconductor a candidate for the carrier management gate layer in the AC-OEL. As an external electric field is applied, pointing from the ITO side to the metal anode, the "forward" direction of the devicediode, the positive hole carriers drift toward the low work function metal electrode through the $\mathrm{MoO}_{3}$ buffer layer. Simultaneously, the excess electrons trapped in the HGL are transferred by the strong electric field toward the energy barrier at the HGL/Gate interface and tunnel into gate's LUMO level. Electron extraction in the HGL dramatically facilitates the electron hopping rate from poly-TPD to F4TCNQ, resulting in a larger population of holes. In other words, the gate layer "opens the door" for electron extraction. Conversely, when the applied AC voltage changes polarity, and the electrical field is reversed across the entire device, the Fermi level tilts the opposite way around. Holes in the HOMO of poly-TPD are driven in the opposite direction and are accumulated at the interface of gate and HGL because of the high tunneling barrier. The gate thus, "shuts the door" for electron extraction before the next forward cycle arrives. Therefore, the PFN-Br gate plays a role of electron extraction layer ("gate on") in the forward bias and works as dielectric layer ("gate off") in the reverse cycle. 


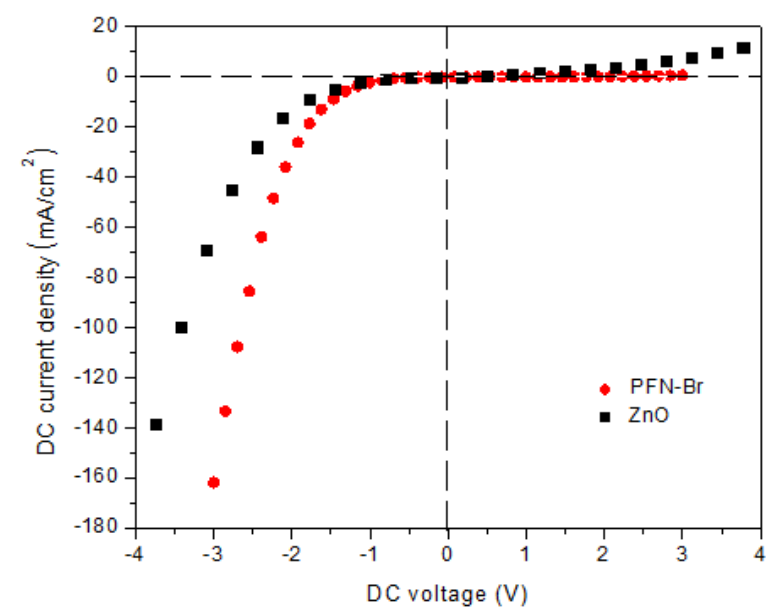

Figure 1 Current-voltage demonstration of $\mathrm{PFN}-\mathrm{Br}$ polymeric gate and $\mathrm{ZnO}$ gate.

\subsection{Flexibility of gated AC-OEL}

The improved flexibility of PFN-Br polymeric layer enormously advances the inorganic gate layer ${ }^{13}$ and allows us to fabricate mechanically flexible gated AC-OEL devices with relatively high performance. The PFN-Br gate was laid down on the cleaned ITO/PET substrate by spin-coating. We employed the doping level of 2:1 of $\operatorname{Ir}(\mathrm{ppy})_{3}$ and $\operatorname{Ir}(\mathrm{MDQ})_{2}(\mathrm{acac})$ in consideration of high luminance and CRI. The flexible structure is shown in Figure 2a. Since the ACOEL device was fabricated on the flexible substrate, the white emission device is able to be bent as shown in Figure $2 \mathrm{~b}$. The SEM image of PFN-Br shows slight line damages with bending in Figure 2c. In comparison, sputtered $\mathrm{ZnO}$ gate exhibits a significant deformation within only few of bending cycles as shown in Figure $2 \mathrm{~d}$.

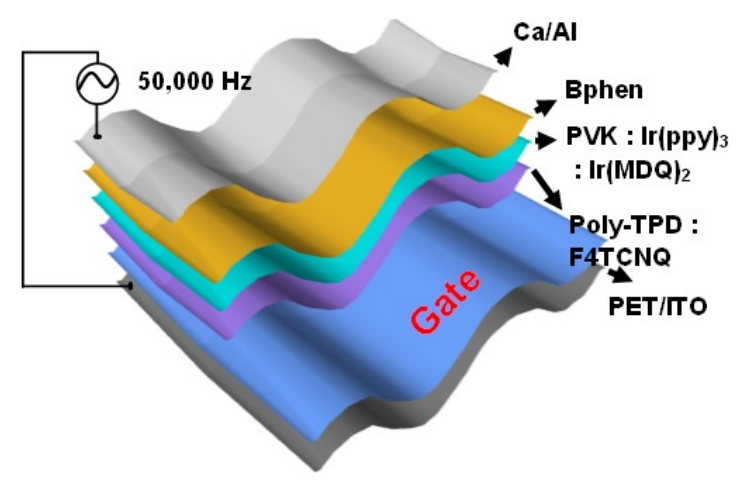

(a)

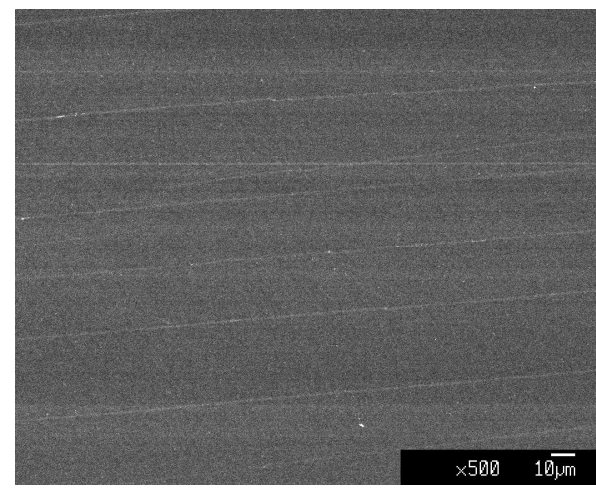

(c)

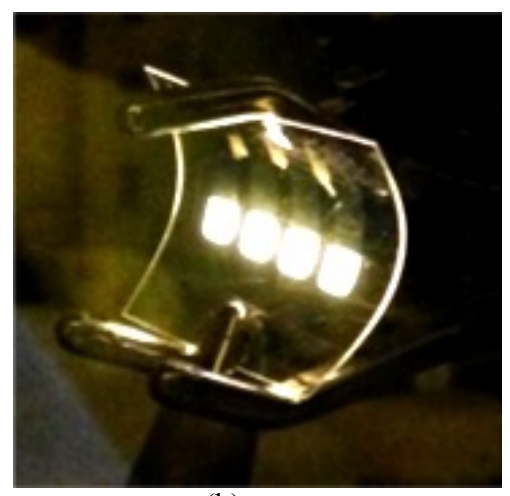

(b)

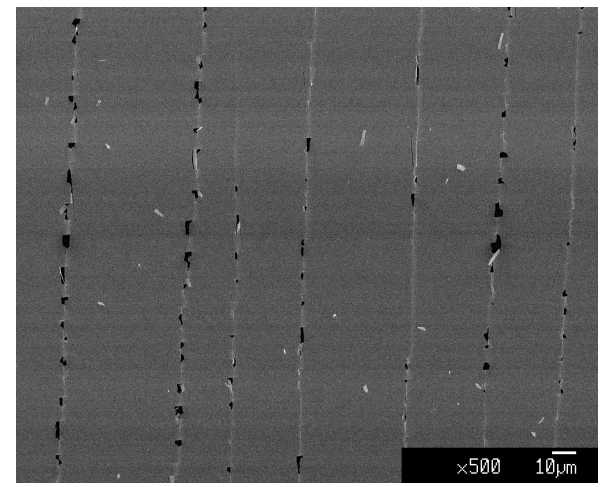

(d)

Figure 2 (a) Flexible device structure of AC-OEL on PET substrate. (b) Demonstration of white emission flexible AC-OEL in bending. SEM images of PFN-Br layer (c) and ZnO layer (d) after same bending cycles. 
The bending angle is defined as negative (from $-90^{\circ}$ to 0 ) in convex bending and as positive (from 0 to $+90^{\circ}$ ) in concave bending. Figure $3 \mathrm{a}$ shows the luminance variation as a function of bending angle which ranges from $-90^{\circ}$ to $+90^{\circ}$ in initial no-bending luminance of $500 \mathrm{~cd} / \mathrm{m}^{2}$ in PFN-Br and $\mathrm{ZnO}$ devices. The PFN-Br gated flexible device shows a relatively stable luminescent output compared with $\mathrm{ZnO}$-gated device. This is attributed to the ITO's tensile deformation on $\mathrm{ZnO}$ layer during bending. In addition, iterative bending tests on the AC-OEL devices were performed on sputtered $\mathrm{ZnO}$ gate and PFN-Br gate devices shown in Figure 3b. The luminance drops less $30 \%$ from the initial in PFN-Br device with 200 bending cycles. In comparison, devices with sputtered $\mathrm{ZnO}$ exhibit a significant luminance degradation (over the half of the initial brightness) within only 5 bending cycles.

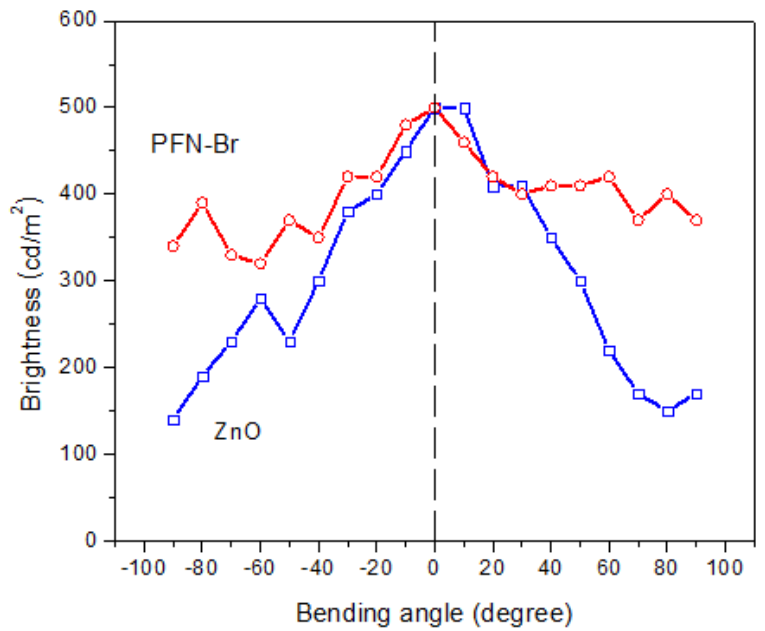

(a)

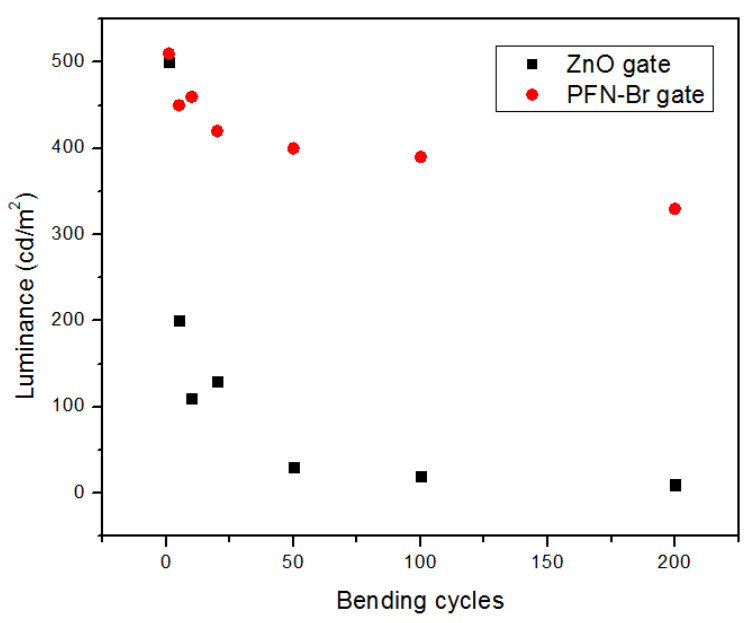

(b)

Figure 3 Bending test of flexible organic AC-EL devices with sputtered $\mathrm{ZnO}$ gate and PFB-Br gate. (a) Luminance as a function of bending angles. (b) Luminance as a function of the number of cycles $(1,5,10,20,50,100$, and 200).

Furthermore, the frequency responses of the flexible AC-OEL device were tested in the flat and bending $\left(90^{\circ}\right)$ positions. The results are shown in Figure 4a where a trivial resonant frequency shift and no current variations were found. Meanwhile, the white EL spectra in Figure 4a is shown to remain intact and stable on color rendering index (CRI) over 81 at $2800 \mathrm{~K}$ color temperature with a $90^{\circ}$ bending.

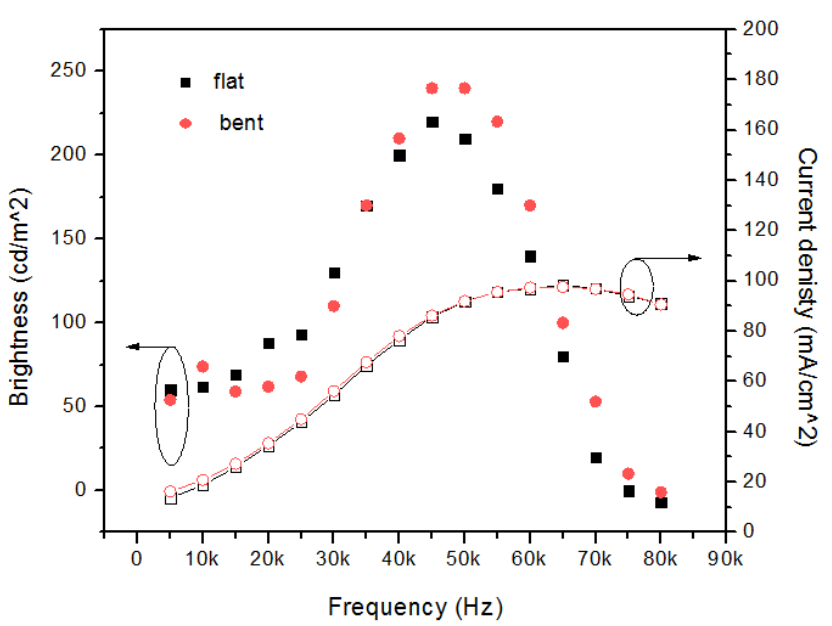

(a)

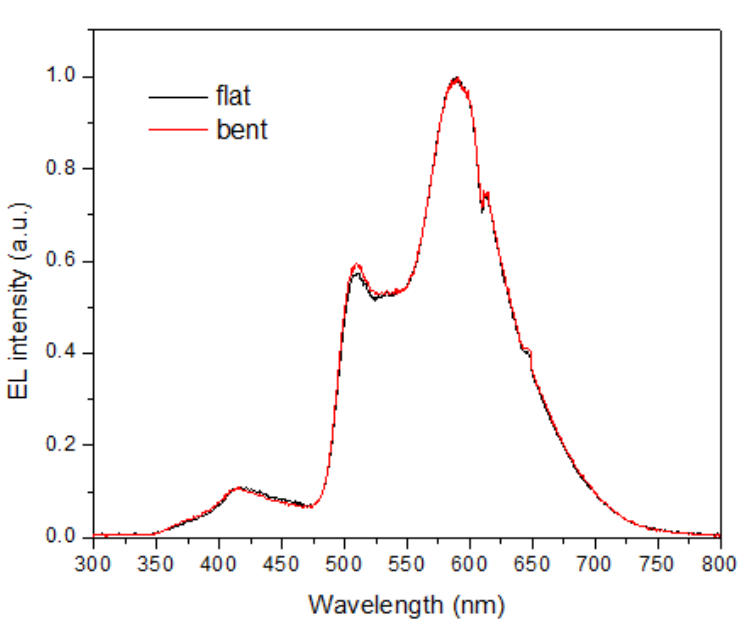

(b)

Figure 4 (a) Device performance over frequency in flat and bending positions. (b) The EL spectrum measurement in flat and bending positions. 


\section{CONCLUSION}

In this work, we showed a flexible gated AC-OEL devices on PET substrates using an n-type conjugate polymer, PFN$\mathrm{Br}$, as carrier gate layer. The small deviation of flexed device electroluminescent parameters demonstrates the superior bending stability of PFN-Br-gated AC-OEL device.

\section{REFERENCES}

[1] Fröbel, M., Schwab, T., Kliem, M., Hofmann, S., Leo, K.., Gather, M. C., "Get it white: color-tunable AC/DC OLEDs," Light Sci. Appl. 4(September 2014), e247 (2015).

[2] Xu, J., Smith, G. M., Dun, C., Cui, Y., Liu, J., Huang, H., Huang, W.., Carroll, D. L., "Layered , Nanonetwork Composite Cathodes for Flexible, High-Efficiency, Organic Light Emitting Devices," Adv. Funct. Mater. 25(28), 4397-4404 (2015).

[3] Micelles, C., Cho, S. H., Jo, S. S., Hwang, I., Sung, J., Seo, J., Jung, S., Bae, I., Choi, J. R., et al., "Extremely Bright Full Color Alternating Current Electroluminescence of Solution-Blended Fluorescent Polymers with SelfAssembled Block," ACS Nano 7(12), 10809-10817 (2013).

[4] Xu, J., Carroll, D. L., Shao, L., Li, P., Dun, C.., Nolan, L., "Polymer Gating White Flexible Field-Induced Lighting Device," Adv. Mater. Technol., Accepted (2017).

[5] Perumal, A., Fröbel, M., Gorantla, S., Gemming, T., Lüssem, B., Eckert, J.., Leo, K., "Novel Approach for Alternating Current (AC)-Driven Organic Light-Emitting Devices," Adv. Funct. Mater. 22(1), 210-217 (2012).

[6] Suzuki, C., Inogochi, T.,, Mito, S., “Thin Film EL Displays,” Inf. Display, SID Int. Symp. 13, 14-19 (1977).

[7] Nanoto, H., Minami, T., Murakami, S.., Takata, S., "Low-voltage-driven ZnS: Mn thin film electroluminescent devices using insulating dielectric ceramic sheets," Thin Solid Films 164, 363-367 (1988).

[8] Chen, Y., Xia, Y., Smith, G. M.., Carroll, D. L., "Frequency-Dependent, Alternating Current-Driven, FieldInduced Polymer Electroluminescent Devices with High Power Efficiency," Adv. Mater. 26, 8133-8140 (2014).

[9] Cho, S. H., Sung, J., Hwang, I., Kim, R. H., Choi, Y. S., Jo, S. S., Lee, T. W.., Park, C., "High performance AC electroluminescence from colloidal quantum dot hybrids.," Adv. Mater. 24(33), 4540-4546 (2012).

[10] Xu, J., Carroll, D. L., Li, P., Nolan, L. V., Shao, L.., Dun, C., "Solution Processing Small-Molecule Organic Emitter in Field-Induced, Carrier Gated Lighting Devices," Adv. Opt. Mater. 5(6), 1600917 (2017).

[11] Xu, J., Carroll, D. L., Smith, G. M., Dun, C.., Cui, Y., "Achieving High Performance in AC-Field Driven Organic Light Sources,” Sci. Rep. 6, 24116, Nature Publishing Group (2016).

[12] Lee, J. H., Cho, S. H., Kim, R. H., Jeong, B., Hwang, S. K., Hwang, I., Kim, K. L., Kim, E. H., Lee, T.-W., et al., "A field-induced hole generation layer for high performance alternating current polymer electroluminescence and its application to extremely flexible devices," J. Mater. Chem. C, Royal Society of Chemistry (2016).

[13] Lee, J., Han, T.-H., Park, M.-H., Jung, D. Y., Seo, J., Seo, H.-K., Cho, H., Kim, E., Chung, J., et al., “Synergetic electrode architecture for efficient graphene-based flexible organic light-emitting diodes," Nat. Commun. 7, 11791, Nature Publishing Group (2016). 